\title{
IN QUEST OF OPTIMAL ALGORITHMS FOR MOLECULAR DYNAMICS SIMULATION OF SURFACE PHENOMENA
}

\author{
J. Mościńsiki, W. Alda, M. Bargiel, M. Bubak, W. Dzwinel, \\ J. Kitowski, E. Mościńska and Z. SkotniczNY \\ Academy of Mining and Metallurgy, Institute of Computer Science \\ Al. Mickiewicza 30, 30-059 Kraków, Poland \\ (Received March 21, 1991)
}

\begin{abstract}
In this paper we present short-range molecular dynamics algorithms and programs elaborated for sequential and vector computer architectures. They are suitable for investigations of surface and subsurface layer properties which require of about $10^{5}$ molecules in a computational box.
\end{abstract}

PACS numbers: 02.70.+d, 64.70.Fx, 68.10.La

\section{Introduction}

The scope of applications of Molecular Dynamics (MD) simulation ranges from the provision of exact data for idealized models of solids, liquids, molecular solutions and macromolecules, to the prediction of properties of models intended to be a realistic representation of natural systems under normal and extreme conditions [1]. Conventionally, the model consists of a number of molecules $(N)$ enclosed into the computational box. Periodic boundary conditions $(p b c)$ are used to make the system pseudo-infinite. A dynamic trajectory of the system is obtained by solving the Newtonian equations of motion at successive timesteps $(\Delta t$; about $10^{-14} \mathrm{~s}$ ), and then thermodynamical, microstructural and transport properties of the model are calculated.

The MD simulation of surface phenomena requires models of about $10^{5}$ molecules due to minimization of the $p b c$ influence on long time and large distance correlations [2]: Calculating forces acting on each molecule is the most CPU time consuming part of each MD simulation ( $95+\%$ of total computing time). Due 
to the order $N^{2}$ for brute all-pair forces computation, this part of each MD algorithm should be carefully optimised. For the same reason MD is one of the main subjects of supercomputer and parallel computers challenge [3].

In the paper we present MD algorithms and programs elaborated for the both sequential and vector computer architectures, and some application results.

\section{Sequential MD algorithms and programs}

We have started with a $\mathrm{C}$ language MD program using the link-cell method [4]. The program can run on mainframes as well as on microcomputers and it was used as a vehicle for testing new algorithms of force calculations.

First, for reduction of the computer time, the cube concept and tiny-cell technique were used. They both differ from the original link-cell method in some additional conditions diminishing the number of candidates for neighbours of each particle [4].

Next, the fixed point arithmetics was applied for the interparticle distance evaluation [5]. Errors introduced by the method were estimated and proved to be on the acceptable level while the main advantage of the method was an increase of calculational efficiency.

Another algorithm, the method of lights, is a sequential version of the CYBER 205 vector oriented one. It gives an easy way to determine neighbours of a given particle, but it has, however, the computational complexity $O\left(N^{5 / 3}\right)$ while the link-cell method is of $O(N)$. In the combined method - the sorted linked list algorithm - the neighbouring molecules are found out as in the link-cell one and next some of them are removed on the base of the method of lights. The sorted linked list MD program [6] is twice faster than the link-cell one.

We have also developed the checker board periodic boundary conditions which enable one to choose different computational boxes (e.g. truncated octahedron, rhombic dodecahedron, etc.) by changing only the size of the basic computational box. Non-cubic boxes make possible to observe relatively long wavelength phenomena using reasonable $N$ [7].

Besides algorithms suitable for molecules interacting with the short range Lennard-Jones (L-J) potentials, we have also elaborated MD programs for ionic systems using the Ewald approximation for long range interactions [8].

The MD programs have been tested on a variety of microcomputers: IBM PC/XT-AT, AT-386/387, AT-486, and microprocessors: Transputer T800 and Motorola $68020 / 68881$. The execution time for argon/krypton mixture and the cutoff radius $2.5 \sigma_{\mathrm{Kr}}$ using the link-cell method is about $3.5 \times 10^{-3} \mathrm{~s}$ per timestep and particle (on T800).

\section{Vector MD algorithms and programs}

We have elaborated three algorithms for short-range MD simulation, suitable for vector processing facilities. Their essential feature are original geometric partitions of the computational box instead of nearest neighbours arrays. 
The first algorithm (pipe method) [9] concerns simulation of dynamics of particles confined in a long cylinder. $P b c$ are introduced along the cylinder axis only (in $z$-direction). The algorithm is based on the observation that in fluid of uniform density the number of neighbours interacting with a given molecule (NCUT) is nearly constant. Particles in the cylinder are sorted due to their $Z$ coordinates and the index vector is set up to returning to original particles indices. $X$ and $Y$ particle coordinates are gathered due to the index vector. For forces calculation the computational cylinder is stepwise shifted in respect to its copy to subsequent neighbouring particles from 1 to NCUT. In this way the forces calculations are fully vectorized.

The strip method [10], [11] is an extension of the previous one to the 3-D bulk system with classical $p b c$. The computational box is divided in $(x-y)$ plane into the elongated in $z$-direction box fragments (strips). The particles in every strip are sorted due to their $Z$ coordinates. The forces calculations are performed in two steps: first forces between particles in the same strip are calculated with the method used for the long cylinder, and next forces between particles from adjacent strips are determined. In the second step every strip is successively shifted in respect to its neighbour.

In the third algorithm (bulk shift method) [10], [11] particles of the 3-D bulk system are mapped on a computational grid in such a way, that every grid point is occupied by only one particle at the most. NCUT is mapping the cutoff radius into number of neighbouring grid points in each direction. The base grid is extended with NCUT points in all directions. The mutual interactions between particles are calculated by stepwise shifting of the computational box in respect to its extended copy in every direction at a distance determined by NCUT.

\section{Applications}

The sequential MD programs have been used for 3-D simulation of particles transitions from the liquid layer to the vapour one. The computational model consists of about several thousands of L-J monoatomic molecules initially arranged on the $f c c$ lattice in a part of computational box below the assumed boundary between the liquid and vapour phases. During the simulation the empty part of the box is being continuously filled with the particles promoted to the vapour phase. The simulation program is supported with a visualization one for graphical presentation of trajectories of particles and histories of the box fragments [12].

Another application of the elaborated algorithms is microscale hydrodynamics for which MD simulation is a relatively recent development [13]. Traditionally the Navier-Stokes equations are applied, but for flows under extreme conditions or for study of what really happens inside a boundary layer, MD may prove to be the only viable approach. Using 2-D molecular dynamics approach we have modelled formation and shedding of vortices during the L-J particle flow around an obstacle [14]. During the simulation the particle trajectories, momenta distribution and local density have been analysed. 


\section{Conclusions}

A set of MD algorithms and programs for the both sequential and vector processing has been developed. The obtained timings for sequential programs are not prohibitively high making possible serious scientific MD simulations of surface phenomena on modern microcomputers. For more realistic models the vector MD programs may be used on the supercomputers and on the future microprocessors [11]. Preliminary results of computer experiments of melting and microscale hydrodynamics account for further investigations.

It would be profitable to develop parallel MD simulation programs due to MD inherent parallelism and availability of parallel architectures on microcomputers.

\section{Acknowledgements}

We are grateful to Professor Jerzy Kapelewski for arranging partial financial support under the Project CPBP 01.08.

\section{References}

[1] Simulation of Liquids and Solids. Molecular Dynamics and Monte Carlo Methods in Statistical Mechanics, Eds. G. Ciccotti, D. Frenkel, I.R. McDonald, North-Holland, Amsterdam 1987.

[2] V. Rosato, G. Ciccotti, V. Pontikis, Phys. Rev. B 33, 1860 (1986).

[3] D. Fincham, Mol. Simul. 1, 1 (1987).

[4] M. Bargiel, W. Dzwinel, J. Kitowski, J. Mościński, Comput. Phys. Commun. 64, 193 (1991).

[5] W. Dzwinel, M. Bargiel, J. Kitowski, J. Mościński, Mol. Simul. 5, 383 (1991).

[6] W. Dzwinel, M. Bargieł, J. Kitowski, J. Mościński, Mol. Simul. 4, 229 (1989).

[7] W. Dzwinel, J. Kitowski, J. Mościński, Mol. Simul., in print.

[8] W. Alda, J. Mościński, in: Proc. of Polar Solids Discussion Group Meeting on Ion Transport in Solids, Mansfield College, Oxford 1990.

[9] J. Mościński, J. Kitowski, Z.A. Rycerz, P.W.M. Jacobs, Comput. Phys. Commun. 54, 47 (1989).

[10] J. Mościński, M. Bargieł, J. Kitowski, Z. Skotniczny, Z.A. Rycerz, P.W.M. Jacobs, in: Proc. of Internat. Conf. on Supercomputing, Crete 1989, Eds. D. Gannon, E. Houstis, ACM Publications, Baltimore.

[11] J. Kitowski, J. Mościński, Mol. Simul., in print.

[12] M. Bubak, R. Słota, submitted to Comput. Phys. Commun.

[13] D.C. Rapaport, Phys. Rev. A, 36, 3288 (1987).

[14] J. Tarbaj, M. Zimnoch, M. Bubak, J. Kitowski, J. Mościński, CCP5 Information Quarterly for Computer Simulation of Condensed Phases 33, 62 (1991) (informal Newsletter of Daresbury Laboratory, Warrington, England). 